\title{
Use and Costs of Medical Foods and Convenience-Packaged Drugs in the California Workers' Compensation System
}

\author{
Tracy Kuo Lin, MSc, PhD, and Leslie Wilson, PhD
}

\begin{abstract}
BACKGROUND: Sales of medical foods (MFs) and convenience packages (CPs) are projected to exceed $\$ 2$ billion in the United States, with an annual growth rate of $10 \%$. Several studies have highlighted the rapid growth of MF use within the California Workers' Compensation System (CAWCS). To curb this growth, California implemented Assembly Bill 378 (AB 378) in 2012 to regulate physicians' incentives to dispense MFs and CPs. AB 378's regulation on only physician-dispensed MFs and CPs and not pharmacydispensed MFs and CPs generated a setting for evaluating the differential effect of the bill on MF and CP use and cost.
\end{abstract}

OBJECTIVES: To (a) examine the use and cost of MFs and CPs in the CAWCS that are not for inborn errors of metabolism and (b) evaluate the regulatory effect of $A B 378$.

METHODS: This study adopted a quantitative approach and employed descriptive statistics and t-tests. The analyses used the most recent complete annual claims data from the Workers' Compensation Information System dataset to evaluate MF and CP claims frequencies and dollar amounts reimbursed from 2011 to 2013 and to compare the difference between physician-dispensed and pharmacy-dispensed products.

RESULTS: Of 151,107 MFs and CPs billed, 95,528 (63.2\%) prescriptions were reimbursed. The reimbursed MFs and CPs accounted for approximately $\$ 19$ million paid to pharmacies and physicians over 3 years. Physiciandispensed MFs, which were regulated by AB 378 in January 2012, experienced a reduction in mean amount reimbursed by $\$ 9.95(P<0.001)$-from $\$ 195.64$ to \$185.68 - compared with the mean amount reimbursed in 2011. Conversely, physician-dispensed CPs, as well as pharmacy-dispensed MFs and CPs, did not experience a decrease in mean amount reimbursed.

CONCLUSIONS: The results indicated that $A B 378$ was associated with a statistically significant reduction in physician-dispensed MFs. Concomitantly, the results from t-tests showed no statistically significant difference in mean amount reimbursed for MFs and CPs to pharmacies before and after $A B$ 378. The finding was expected and as hypothesized because $A B 378$ did not regulate pharmacy-dispensed MFs and CPs. Legislative measures, such as AB 378 in California, may influence rising costs and use of MFs and CPs in general. Other workers' compensation systems could adopt similar legislation to affect the behavior of physician prescribing of non-inborn errors of metabolism MFs and further test these findings.

J Manag Care Spec Pharm. 2018;24(8):819-33

Copyright @2018, Academy of Managed Care Pharmacy. All rights reserved.

\section{What is already known about this subject}

Medical foods (MFs) often have inadequate scientific support, and public data on the type and number of MFs used and available on the U.S. market today are lacking.

Despite the absence of data and scientific evidence, insurance claims for MFs - specifically those not for inborn errors of metabolism-are rising.

\section{What this study adds}

This study examined the use and cost of MFs and convenience packages (CPs) in the California Workers' Compensation System, which has not been considered since Assembly Bill 378 (AB 378) went into effect on January 1, 2012.

The most frequently reimbursed MFs and CPs were for indications of insomnia and pain, with new MFs quickly gaining market share. T-tests demonstrated that the difference in amount reimbursed for MFs before and after the implementation of AB 378 was statistically significant, providing support for the effectiveness of AB 378 in regulating MF use.

$\mathrm{T}$ he availability of medical foods (MFs) is expanding, and increasingly MFs are offered as a treatment choice without evidence of their safety or efficacy. An MF is "a food which is formulated to be consumed or administered enterally under the supervision of a physician and which is intended for the specific dietary management of a disease or condition for which distinctive nutritional requirements, based on recognized scientific principles, are established by medical evaluation." A convenience-packaged MF (co-pack [CP]) is a generic drug packaged with an MF.

MFs and CPs are dispensed directly from a physician's office or from a pharmacy. MFs are not a food or drug or supplement, but something in between (see list of MFs in Table 1). At the federal level, MFs are regulated partly as a food, but one that requires scientific evidence and a distinct medical need, and partly as a drug, but one that does not require clinical trial evidence for efficacy and safety for approval by the U.S. Food and Drug Administration (FDA). Consequently, many of the MFs on the market lack evidence to support their safety and efficacy. ${ }^{2}$ While state-level actors, such as workers' compensation (WC) systems, can regulate the use of MFs, as many as 
45 out of the 50 state WC systems do not have specific regulations on payment for MFs and CPs.

Sales for MFs are projected to exceed $\$ 2$ billion in the United States, with an annual growth rate of $10 \%{ }^{3}$ Several studies have highlighted the rapid growth of $\mathrm{MF}$ and $\mathrm{CP}$ use within the California Workers' Compensation System (CAWCS), suggesting the importance of tracking their reimbursement mechanisms and amounts. ${ }^{4,5}$ MFs are indispensable for individuals with inborn errors of metabolism (IEMs) —an inherited biochemical disorder in which a specific enzyme defect interferes with metabolism of protein, fat, or carbohydrate. However, the documented increase in MF use within CAWCS is for MFs not used for IEM-despite the absence of data and scientific evidence- and thus deserves assessment of their use and costs. ${ }^{6}$

CAWCS currently uses the Centers for Medicare \& Medicaid Services (CMS) weekly National Drug Code (NDC) lowest price list to benchmark prices for covered drugs. However, this system excludes MFs, leaving CMS without benchmark prices for MFs or CPs. This lack of pricing guidance creates a financial incentive for physicians, who legally are authorized to dispense pharmaceutical products in WC systems, to prescribe non-IEM MFs, and may play a role in the documented growth in MF use. To curb this growth, California passed Assembly Bill 378 (AB 378) in 2011 and implemented the bill in 2012 to regulate physicians' incentives. ${ }^{7}$

The combination of the growing use of only non-IEM MFs in CAWCS and the new regulation makes CAWCS an ideal sample for studying MF and CP use. No study has examined comprehensively the use and cost of MFs and CPs in CAWCS since AB 378 came into effect on January 1, 2012.

The main objectives of this study were to (a) examine the use and cost of non-IEM MFs and CPs in CAWCS and (b) evaluate the regulatory effect of AB 378. Specifically, employing the CAWCS claims dataset, the first aim was to determine the specific names of non-IEM MFs reimbursed; if the use of non-IEM MFs and CPs was increasing between 2011 and 2013; and if there were any changes in their cost during that time frame. The second aim was to evaluate if $A B 378$ influenced the frequency of claims and reimbursed amounts of non-IEM MFs and CPs, especially for the physician-dispensed goods, which AB 378 targets, and compared with the pharmacy-dispensed MFs and CPs, which were not targeted.

\section{Methods}

\section{Data}

The research design and data use were approved by the institutional review board of the University of California, San Francisco. The analyses employed the most recent complete annual claims data from the Workers' Compensation Information System (WCIS) dataset from CAWCS to evaluate MF and CP claims frequencies and dollar amounts reimbursed from 2011 to 2013.
The unit of analysis (each row) for the database was by prescription claimed, and the database contained the following variables: claim identifiers, billing identifiers, paid (reimbursed) amounts in U.S. dollars, service adjustment codes, drug names, NDC numbers, service dates, and quantity dispensed (days covered) for each billed pharmaceutical product. Each claim represented a single prescription. Each patient in the CAWCS was assigned a unique claim identifier, and each unique bill was identified as either pharmacy or physician dispensed. Service adjustment codes indicated reasons for discrepancy between billed and paid (reimbursed) amounts; the variable allowed us to categorize each claim line (representing a unique prescription) into reimbursed, denied, adjusted, or duplicate bill. Duplicates and negative paid amounts were removed from the dataset.

A specific procedure was followed to identify MFs and CPs in our dataset. An MF should not have an NDC number, with FDA guidance stating that the presence of an NDC number on a food product misbrands the product. ${ }^{1}$ Despite the guidance, all the MFs and CPs in the WCIS dataset were designated with an NDC number, which is required for reimbursement.

This study therefore identified a prescription as an MF or a CP claim in the WCIS dataset by examining (a) the NDC description online, (b) the product website, (c) the package insert, and (d) the RED BOOK description. During the identification process, MF ingredients and MF indications were also evaluated; all MFs and CPs in the database were non-IEM MFs and CPs, as they were prescribed to working adults and related to a work injury.

The main policy intervention was statute $\mathrm{AB} 378,{ }^{7}$ because one of its legislative intents was to address the increasing use of MFs and CPs through (a) implementing fee schedules limiting payment amount and (b) regulating physicians' financial incentives when prescribing and dispensing MFs and CPs.

\section{Analysis}

The analyses for physician-dispensed and pharmacy-dispensed MFs and CPs claims were conducted separately because physician-dispensed MFs and CPs were regulated by AB 378 starting in January 2012, while pharmacy-dispensed MFs and $\mathrm{CPs}$ were not specifically regulated by $\mathrm{AB} 378$. The results provided $\mathrm{MF}$ and $\mathrm{CP}$ claims frequencies and descriptive statistics on paid costs; i.e., reimbursed amount, including frequencies, means, and medians.

T-tests were employed first to determine if there was a statistically significant difference in the mean amount reimbursed per claim for physician-dispensed MFs and CPs before (January 1, 2011-December 31, 2011) and after (January 1, 2012-December 31, 2013) the implementation of AB 378. This study hypothesized that there should be a significant difference, as AB 378 specifically regulated physician-dispensed MFs and CPs. The second t-test evaluated if there was a 


\begin{tabular}{|c|c|c|c|c|c|c|}
\hline Medical Foods ${ }^{\mathrm{a}}$ & Indication $^{\mathrm{b}}$ & $\begin{array}{l}\text { Prescriptions } \\
\text { Reimbursedc }\end{array}$ & $\begin{array}{c}\text { Percentage } \\
\text { Reimbursed }\end{array}$ & $\begin{array}{c}\text { Total Amount } \\
\text { Reimbursed, } \\
\$ \mathrm{~s}\end{array}$ & $\begin{array}{c}\text { Mean Amount } \\
\text { Reimbursed, } \\
\$^{f}\end{array}$ & $\begin{array}{c}\text { Standard } \\
\text { Deviationg }\end{array}$ \\
\hline \multicolumn{7}{|c|}{ Dispensed by Physicians } \\
\hline \multicolumn{7}{|c|}{$2011-2013$} \\
\hline Theramine & Chronic low back pain/inflammation & 34,960 & 48.52 & $8,539,928.95$ & 244.28 & 243.44 \\
\hline Sentra PM & Insomnia & 12,733 & 17.67 & $1,628,585.24$ & 127.90 & 122.30 \\
\hline Sentra AM & Insomnia & 7,396 & 10.26 & $999,937.92$ & 135.20 & 167.46 \\
\hline GABAdone & Insomnia & 5,687 & 7.89 & $763,457.23$ & 134.25 & 111.93 \\
\hline Trepadone & Joint disorder pain/inflammation & 2,909 & 4.04 & $786,722.58$ & 270.44 & 269.04 \\
\hline Proteolin & Joint disorder pain/inflammation & 2,635 & 3.66 & $340,316.26$ & 129.15 & 84.92 \\
\hline Somnicin & Insomnia & 1,988 & 2.76 & $196,663.86$ & 98.93 & 135.25 \\
\hline Apptrim & Obesity & 1,810 & 2.51 & $293,289.50$ & 162.04 & 173.22 \\
\hline Sintralyne-PM & Insomnia & 744 & 1.03 & $42,465.08$ & 57.08 & 49.85 \\
\hline Limbrel & Osteoarthritis & 354 & 0.49 & $44,081.14$ & 124.52 & 152.87 \\
\hline Hypertensa & Hypertension & 260 & 0.36 & $33,493.29$ & 128.82 & 192.95 \\
\hline Deplin & Major depression & 170 & 0.24 & $17,773.79$ & 104.55 & 68.59 \\
\hline L-Methylfolate & Depression, anemia, renal impairment & 101 & 0.14 & $7,010.21$ & 69.41 & 43.24 \\
\hline Metanx & Neuropathic pain & 66 & 0.09 & $4,055.11$ & 61.44 & 56.22 \\
\hline Folbic & Atherosclerosis & 65 & 0.09 & $1,415.09$ & 21.77 & 17.83 \\
\hline Percura & Peripheral neuropathy & 53 & 0.07 & $14,188.70$ & 267.71 & 257.27 \\
\hline Foltx & Hyperhomocysteinemia & 35 & 0.05 & $1,350.55$ & 38.59 & 11.92 \\
\hline Folbee & Kidney failure & 22 & 0.03 & 530.48 & 24.11 & 7.97 \\
\hline Foltanx & Diabetic neuropathy & 14 & 0.02 & $1,064.32$ & 76.02 & 3.72 \\
\hline Virilex & Fertility & 10 & 0.01 & 777.29 & 77.73 & 61.89 \\
\hline Cerefolin & Cognitive impairment, hyperhomocysteinemia & 9 & 0.01 & 565.65 & 62.85 & 59.62 \\
\hline Ferrex & Iron deficiency, anemia & 8 & 0.01 & 109.60 & 13.70 & 0.00 \\
\hline FABB & Nutrition in cystic fibrosis & 5 & 0.01 & 201.98 & 40.40 & 17.39 \\
\hline Ferocon & Anemia & 4 & 0.01 & 103.48 & 25.87 & 4.78 \\
\hline Glucerna & Impaired glucose tolerance & 4 & 0.01 & 405.27 & 101.32 & 151.20 \\
\hline VSL\#3 & Ulcerative colitis & 4 & 0.01 & 71.20 & 17.80 & 14.30 \\
\hline Enlyte & Depression & 3 & 0.00 & 304.41 & 101.47 & 0.00 \\
\hline Pulmona & Pulmonary hypertension & 3 & 0.00 & 670.52 & 223.51 & 78.53 \\
\hline Folcaps & Digestive disorders & 2 & 0.00 & 33.80 & 16.90 & 0.00 \\
\hline \multicolumn{2}{|l|}{ Total } & 72,054 & 100.00 & $13,719,572.50$ & 190.41 & 185.10 \\
\hline \multicolumn{7}{|l|}{2011} \\
\hline Theramine & Chronic low back pain/inflammation & 18,580 & 54.34 & $4,406,459.21$ & 237.16 & 226.42 \\
\hline Sentra PM & Insomnia & 5,958 & 17.43 & $765,383.15$ & 128.46 & 124.77 \\
\hline Sentra AM & Insomnia & 3,759 & 10.99 & $550,222.97$ & 146.37 & 189.50 \\
\hline GABAdone & Insomnia & 2,632 & 7.70 & $346,742.30$ & 131.74 & 113.32 \\
\hline Trepadone & Joint disorder pain/inflammation & 1,275 & 3.73 & $370,477.43$ & 290.57 & 297.52 \\
\hline Apptrim & Obesity & 797 & 2.33 & $125,567.87$ & 157.55 & 164.45 \\
\hline Proteolin & Joint disorder pain/inflammation & 591 & 1.73 & $60,997.62$ & 103.21 & 83.00 \\
\hline Limbrel & Osteoarthritis & 228 & 0.67 & $33,543.56$ & 147.12 & 177.85 \\
\hline Sintralyne-PM & Insomnia & 166 & 0.49 & $14,069.79$ & 84.76 & 59.72 \\
\hline Hypertensa & Hypertension & 59 & 0.17 & $8,836.19$ & 149.77 & 165.73 \\
\hline Deplin & Major depression & 46 & 0.13 & $3,176.58$ & 69.06 & 49.16 \\
\hline Metanx & Neuropathic pain & 34 & 0.10 & $1,439.22$ & 42.33 & 46.33 \\
\hline Folbic & Atherosclerosis & 33 & 0.10 & 445.77 & 13.51 & 15.05 \\
\hline Foltx & Hyperhomocysteinemia & 15 & 0.04 & 593.47 & 39.56 & 17.75 \\
\hline L-Methylfolate & Depression, anemia, renal impairment & 6 & 0.02 & 393.06 & 65.51 & 0.00 \\
\hline Virilex & Fertility & 4 & 0.01 & 277.44 & 69.36 & 80.09 \\
\hline Cerefolin & Cognitive impairment, hyperhomocysteinemia & 3 & 0.01 & 0.00 & 0.00 & 0.00 \\
\hline FABB & Nutrition in cystic fibrosis & 2 & 0.01 & 42.92 & 21.46 & 0.00 \\
\hline Pulmona & Pulmonary hypertension & 2 & 0.01 & 492.35 & 246.18 & 96.17 \\
\hline
\end{tabular}


TABLE 1 Medical Foods by Dispenser from 2011 to 2013 (continued)

\begin{tabular}{|c|c|c|c|c|c|c|}
\hline Medical Foods ${ }^{a}$ & Indication $^{\mathrm{b}}$ & $\begin{array}{l}\text { Prescriptions } \\
\text { Reimbursed }^{c}\end{array}$ & $\begin{array}{c}\text { Percentage } \\
\text { Reimbursed }^{\mathrm{d}}\end{array}$ & $\begin{array}{c}\text { Total Amount } \\
\text { Reimbursed, } \\
\$ \text { \$e }\end{array}$ & $\begin{array}{c}\text { Mean Amount } \\
\text { Reimbursed, } \\
\$^{f}\end{array}$ & $\begin{array}{c}\text { Standard } \\
\text { Deviationg }\end{array}$ \\
\hline \multicolumn{7}{|l|}{2011} \\
\hline Folbee & Kidney failure & 1 & 0.00 & 24.33 & 24.33 & - \\
\hline VSL\#3 & Ulcerative colitis & 1 & 0.00 & 2.15 & 2.15 & - \\
\hline Total & & 34,192 & 100.00 & $6,689,187.38$ & 195.64 & 206.96 \\
\hline \multicolumn{7}{|l|}{2012} \\
\hline Theramine & Chronic low back pain/inflammation & 9,629 & 47.00 & $2,424,080.55$ & 251.75 & 232.89 \\
\hline Sentra PM & Insomnia & 3,372 & 16.46 & $461,942.07$ & 136.99 & 117.40 \\
\hline Proteolin & Joint disorder pain/inflammation & 1,718 & 8.39 & $233,257.74$ & 135.77 & 82.94 \\
\hline Sentra AM & Insomnia & 1,585 & 7.74 & $212,144.28$ & 133.84 & 143.51 \\
\hline GABAdone & Insomnia & 1,567 & 7.65 & $233,444.38$ & 148.98 & 110.67 \\
\hline Trepadone & Joint disorder pain/inflammation & 935 & 4.56 & $248,600.52$ & 265.88 & 247.57 \\
\hline Apptrim & Obesity & 608 & 2.97 & $108,608.62$ & 178.63 & 176.83 \\
\hline Somnicin & Insomnia & 411 & 2.01 & $50,246.11$ & 122.25 & 136.17 \\
\hline Sintralyne-PM & Insomnia & 355 & 1.73 & $19,514.22$ & 54.97 & 42.70 \\
\hline Hypertensa & Hypertension & 76 & 0.37 & $10,397.97$ & 136.82 & 198.87 \\
\hline Deplin & Major depression & 75 & 0.37 & $7,197.31$ & 95.96 & 50.78 \\
\hline Limbrel & Osteoarthritis & 65 & 0.32 & $4,831.72$ & 74.33 & 100.71 \\
\hline L-Methylfolate & Depression, anemia, renal impairment & 33 & 0.16 & $2,303.98$ & 69.82 & 43.21 \\
\hline Foltx & Hyperhomocysteinemia & 13 & 0.06 & 459.64 & 35.36 & 3.62 \\
\hline Folbee & Kidney failure & 11 & 0.05 & 264.18 & 24.02 & 1.04 \\
\hline Metanx & Neuropathic pain & 11 & 0.05 & $1,349.96$ & 122.72 & 27.72 \\
\hline Folbic & Atherosclerosis & 8 & 0.04 & 202.96 & 25.37 & 17.52 \\
\hline Ferrex & Iron deficiency, anemia & 6 & 0.03 & 82.20 & 13.70 & 0.00 \\
\hline FABB & Nutrition in cystic fibrosis & 2 & 0.01 & 109.20 & 54.60 & 0.00 \\
\hline Folcaps & Digestive disorders & 2 & 0.01 & 33.80 & 16.90 & 0.00 \\
\hline VSL\#3 & Ulcerative colitis & 2 & 0.01 & 32.20 & 16.10 & 0.00 \\
\hline Glucerna & Impaired glucose tolerance & 1 & 0.00 & 319.95 & 319.95 & - \\
\hline Pulmona & Pulmonary hypertension & 1 & 0.00 & 178.17 & 178.17 & - \\
\hline Total & & 20,486 & 100.00 & $4,019,601.73$ & 196.21 & 197.11 \\
\hline \multicolumn{7}{|l|}{2013} \\
\hline Theramine & Chronic low back pain/inflammation & 6,751 & 38.85 & $1,709,389.19$ & 253.21 & 256.68 \\
\hline Sentra PM & Insomnia & 3,403 & 19.58 & $401,260.02$ & 117.91 & 122.06 \\
\hline Sentra AM & Insomnia & 2,052 & 11.81 & $237,570.67$ & 115.78 & 137.09 \\
\hline Somnicin & Insomnia & 1,577 & 9.08 & $146,417.75$ & 92.85 & 134.38 \\
\hline GABAdone & Insomnia & 1,488 & 8.56 & $183,270.55$ & 123.17 & 109.21 \\
\hline Trepadone & Joint disorder pain/inflammation & 699 & 4.02 & $167,644.63$ & 239.83 & 237.24 \\
\hline Apptrim & Obesity & 405 & 2.33 & $59,113.01$ & 145.96 & 182.63 \\
\hline Proteolin & Joint disorder pain/inflammation & 326 & 1.88 & $46,060.90$ & 141.29 & 89.39 \\
\hline Sintralyne-PM & Insomnia & 223 & 1.28 & $8,881.07$ & 39.83 & 43.36 \\
\hline Hypertensa & Hypertension & 125 & 0.72 & $14,259.13$ & 114.07 & 201.32 \\
\hline L-Methylfolate & Depression, anemia, renal impairment & 62 & 0.36 & $4,313.17$ & 69.57 & 45.65 \\
\hline Limbrel & Osteoarthritis & 61 & 0.35 & $5,705.86$ & 93.54 & 36.81 \\
\hline Percura & Peripheral neuropathy & 53 & 0.31 & $14,188.70$ & 267.71 & 257.27 \\
\hline Deplin & Major depression & 49 & 0.28 & $7,399.90$ & 151.02 & 82.45 \\
\hline Folbic & Atherosclerosis & 24 & 0.14 & 766.36 & 31.93 & 16.29 \\
\hline Metanx & Neuropathic pain & 21 & 0.12 & $1,265.93$ & 60.28 & 60.49 \\
\hline Foltanx & Diabetic neuropathy & 14 & 0.08 & $1,064.32$ & 76.02 & 3.72 \\
\hline Folbee & Kidney failure & 10 & 0.06 & 241.97 & 24.20 & 12.13 \\
\hline Foltx & Hyperhomocysteinemia & 7 & 0.04 & 297.44 & 42.49 & 0.87 \\
\hline Cerefolin & Cognitive impairment, hyperhomocysteinemia & 6 & 0.03 & 565.65 & 94.28 & 46.19 \\
\hline Virilex & Fertility & 6 & 0.03 & 499.85 & 83.31 & 54.34 \\
\hline Ferocon & Anemia & 4 & 0.02 & 103.48 & 25.87 & 4.78 \\
\hline Enlyte & Depression & 3 & 0.02 & 304.41 & 101.47 & 0.00 \\
\hline
\end{tabular}


TABLE 1 Medical Foods by Dispenser from 2011 to 2013 (continued)

\begin{tabular}{|c|c|c|c|c|c|c|}
\hline Medical Foods ${ }^{a}$ & Indication $^{\mathrm{b}}$ & $\begin{array}{l}\text { Prescriptions } \\
\text { Reimbursed }^{c}\end{array}$ & $\begin{array}{l}\text { Percentage } \\
\text { Reimbursed }^{\mathrm{d}}\end{array}$ & $\begin{array}{c}\text { Total Amount } \\
\text { Reimbursed, } \\
\$ \text { e }\end{array}$ & $\begin{array}{c}\text { Mean Amount } \\
\text { Reimbursed, } \\
\$^{f}\end{array}$ & $\begin{array}{c}\text { Standard } \\
\text { Deviationg }\end{array}$ \\
\hline \multicolumn{7}{|l|}{2013} \\
\hline Glucerna & Impaired glucose tolerance & 3 & 0.02 & 85.32 & 28.44 & 49.26 \\
\hline Ferrex & Iron deficiency, anemia & 2 & 0.01 & 27.40 & 13.70 & 0.00 \\
\hline FABB & Nutrition in cystic fibrosis & 1 & 0.01 & 49.86 & 49.86 & - \\
\hline VSL\#3 & Ulcerative colitis & 1 & 0.01 & 36.85 & 36.85 & - \\
\hline \multicolumn{2}{|l|}{ Total } & 17,376 & 100.00 & $3,010,783.39$ & 97.97 & 205.05 \\
\hline \multicolumn{7}{|c|}{ Dispensed by Pharmacies } \\
\hline \multicolumn{7}{|c|}{$2011-2013$} \\
\hline Somnicin & Insomnia & 1,000 & 24.99 & $123,731.26$ & 123.73 & 149.63 \\
\hline Theramine & Chronic low back pain/inflammation & 692 & 17.29 & $225,143.43$ & 325.35 & 318.33 \\
\hline Limbrel & Osteoarthritis & 368 & 9.20 & $35,599.65$ & 96.74 & 64.20 \\
\hline Proteolin & Joint disorder pain/inflammation & 309 & 7.72 & $39,346.52$ & 127.34 & 93.16 \\
\hline Deplin & Major depression & 231 & 5.77 & $25,207.79$ & 109.12 & 72.62 \\
\hline L-Methylfolate & Depression, anemia, renal impairment & 200 & 5.00 & $17,398.49$ & 86.99 & 45.88 \\
\hline Sentra PM & Insomnia & 194 & 4.85 & $35,237.05$ & 181.63 & 125.80 \\
\hline GABAdone & Insomnia & 191 & 4.77 & $13,159.21$ & 68.90 & 113.00 \\
\hline Sintralyne-PM & Insomnia & 190 & 4.75 & $4,980.41$ & 26.21 & 45.62 \\
\hline Metanx & Neuropathic pain & 150 & 3.75 & $13,684.29$ & 91.23 & 79.72 \\
\hline Apptrim & Obesity & 106 & 2.65 & $24,467.29$ & 230.82 & 202.81 \\
\hline Trepadone & Joint disorder pain/inflammation & 89 & 2.22 & $28,797.50$ & 323.57 & 326.36 \\
\hline Sentra AM & Insomnia & 82 & 2.05 & $22,723.12$ & 277.11 & 266.20 \\
\hline Folbic & Atherosclerosis & 79 & 1.97 & $2,039.45$ & 25.82 & 19.11 \\
\hline Cerefolin & Cognitive impairment, hyperhomocysteinemia & 48 & 1.20 & $5,249.46$ & 109.36 & 71.28 \\
\hline Foltanx & Diabetic neuropathy & 14 & 0.35 & $1,099.63$ & 78.55 & 0.45 \\
\hline VSL\#3 & Ulcerative colitis & 13 & 0.32 & $1,678.49$ & 129.11 & 126.73 \\
\hline Foltx & Hyperhomocysteinemia & 12 & 0.30 & 173.10 & 14.43 & 15.07 \\
\hline FABB & Nutrition in cystic fibrosis & 10 & 0.25 & 246.60 & 24.66 & 6.75 \\
\hline Ferocon & Anemia & 10 & 0.25 & 239.06 & 23.91 & 4.45 \\
\hline Folcaps & Digestive disorders & 6 & 0.15 & 70.98 & 11.83 & 0.00 \\
\hline Hypertensa & Hypertension & 5 & 0.12 & 712.41 & 142.48 & 318.60 \\
\hline Ferrex & Iron deficiency, anemia & 2 & 0.05 & 126.72 & 63.36 & 54.87 \\
\hline Glucerna & Impaired glucose tolerance & 1 & 0.02 & 164.63 & 164.63 & - \\
\hline \multicolumn{2}{|r|}{ The o o } & 4,002 & 100.00 & $621,276.54$ & 155.24 & 200.72 \\
\hline \multicolumn{7}{|l|}{2011} \\
\hline Theramine & Chronic low back pain/inflammation & 349 & 30.91 & $94,184.25$ & 269.87 & 324.89 \\
\hline Limbrel & Osteoarthritis & 181 & 16.03 & $17,314.88$ & 95.66 & 65.16 \\
\hline Deplin & Major depression & 124 & 10.98 & $12,179.08$ & 98.22 & 79.67 \\
\hline Sentra PM & Insomnia & 97 & 8.59 & $17,618.92$ & 181.64 & 131.95 \\
\hline GABAdone & Insomnia & 81 & 7.17 & $6,298.37$ & 77.76 & 116.35 \\
\hline Apptrim & Obesity & 55 & 4.87 & $9,575.59$ & 174.10 & 187.65 \\
\hline Trepadone & Joint disorder pain/inflammation & 46 & 4.07 & $15,348.01$ & 333.65 & 370.42 \\
\hline Proteolin & Joint disorder pain/inflammation & 39 & 3.45 & $3,043.94$ & 78.05 & 86.04 \\
\hline Metanx & Neuropathic pain & 38 & 3.37 & $1,642.96$ & 43.24 & 39.69 \\
\hline L-Methylfolate & Depression, anemia, renal impairment & 33 & 2.92 & $2,336.20$ & 70.79 & 24.32 \\
\hline Sentra AM & Insomnia & 29 & 2.57 & $8,982.81$ & 309.75 & 174.97 \\
\hline Folbic & Atherosclerosis & 21 & 1.86 & 659.17 & 31.39 & 20.74 \\
\hline Ferocon & Anemia & 10 & 0.89 & 239.06 & 23.91 & 4.45 \\
\hline Folcaps & Digestive disorders & 6 & 0.53 & 70.98 & 11.83 & 0.00 \\
\hline Foltx & Hyperhomocysteinemia & 6 & 0.53 & 173.10 & 28.85 & 0.00 \\
\hline Cerefolin & Cognitive impairment, hyperhomocysteinemia & 5 & 0.44 & 621.77 & 124.35 & 66.01 \\
\hline VSL\#3 & Ulcerative colitis & 4 & 0.35 & 649.02 & 162.26 & 228.98 \\
\hline Hypertensa & Hypertension & 3 & 0.27 & 0.00 & 0.00 & 0.00 \\
\hline Ferrex & Iron deficiency, anemia & 1 & 0.09 & 24.56 & 24.56 & - \\
\hline
\end{tabular}


TABLE 1 Medical Foods by Dispenser from 2011 to 2013 (continued)

\begin{tabular}{|c|c|c|c|c|c|c|}
\hline Medical Foodsa & Indication $^{\mathrm{b}}$ & $\begin{array}{l}\text { Prescriptions } \\
\text { Reimbursed }^{c}\end{array}$ & $\begin{array}{c}\text { Percentage } \\
\text { Reimbursed }^{\mathrm{d}}\end{array}$ & $\begin{array}{c}\text { Total Amount } \\
\text { Reimbursed, } \\
\$^{\mathrm{e}}\end{array}$ & $\begin{array}{c}\text { Mean Amount } \\
\text { Reimbursed, } \\
\$ \$^{f}\end{array}$ & $\begin{array}{c}\text { Standard } \\
\text { Deviationg }\end{array}$ \\
\hline \multicolumn{7}{|l|}{2011} \\
\hline Glucerna & Impaired glucose tolerance & 1 & 0.09 & 164.63 & 164.63 & - \\
\hline \multicolumn{2}{|l|}{ Total } & 1,129 & 100.00 & $191,127.00$ & 169.29 & 231.40 \\
\hline \multicolumn{7}{|l|}{2012} \\
\hline Somnicin & Insomnia & 288 & 20.18 & $31,003.56$ & 107.65 & 133.32 \\
\hline Proteolin & Joint disorder pain/inflammation & 218 & 15.28 & $28,216.50$ & 129.43 & 91.05 \\
\hline Theramine & Chronic low back pain/inflammation & 210 & 14.72 & $73,564.44$ & 350.31 & 237.98 \\
\hline Limbrel & Osteoarthritis & 109 & 7.63 & $9,921.00$ & 91.02 & 66.44 \\
\hline GABAdone & Insomnia & 92 & 6.45 & $4,291.07$ & 46.64 & 94.44 \\
\hline Sintralyne-PM & Insomnia & 90 & 6.31 & $3,740.25$ & 41.56 & 44.11 \\
\hline L-Methylfolate & Depression, anemia, renal impairment & 78 & 5.46 & $7,426.12$ & 95.21 & 48.72 \\
\hline Deplin & Major depression & 70 & 4.91 & $8,840.69$ & 126.30 & 74.47 \\
\hline Metanx & Neuropathic pain & 59 & 4.13 & $5,763.84$ & 97.69 & 70.69 \\
\hline Sentra PM & Insomnia & 55 & 3.86 & $9,969.68$ & 181.27 & 112.85 \\
\hline Folbic & Atherosclerosis & 36 & 2.52 & 877.13 & 24.36 & 21.81 \\
\hline Apptrim & Obesity & 35 & 2.45 & $8,589.13$ & 245.40 & 151.62 \\
\hline Cerefolin & Cognitive impairment, hyperhomocysteinemia & 30 & 2.11 & $3,417.68$ & 113.92 & 65.97 \\
\hline Trepadone & Joint disorder pain/inflammation & 24 & 1.68 & $8,501.40$ & 354.23 & 274.91 \\
\hline Sentra AM & Insomnia & 20 & 1.40 & $4,883.82$ & 244.19 & 279.91 \\
\hline Foltx & Hyperhomocysteinemia & 6 & 0.42 & 0.00 & 0.00 & 21.81 \\
\hline VSL\#3 & Ulcerative colitis & 6 & 0.42 & 541.60 & 90.27 & 49.24 \\
\hline Hypertensa & Hypertension & 1 & 0.07 & 712.41 & 712.41 & - \\
\hline \multicolumn{2}{|l|}{ Total } & 1,427 & 100.00 & $210,260.00$ & 147.00 & 168.35 \\
\hline \multicolumn{7}{|l|}{2013} \\
\hline Somnicin & Insomnia & 712 & 49.24 & $92,727.70$ & 130.24 & 155.36 \\
\hline Theramine & Chronic low back pain/inflammation & 133 & 9.20 & $57,394.74$ & 431.54 & 376.86 \\
\hline Sintralyne-PM & Insomnia & 100 & 6.92 & $1,240.16$ & 12.40 & 42.63 \\
\hline L-Methylfolate & Depression, anemia, renal impairment & 89 & 6.15 & $7,636.17$ & 85.80 & 48.05 \\
\hline Limbrel & Osteoarthritis & 78 & 5.39 & $8,363.77$ & 107.23 & 58.01 \\
\hline Metanx & Neuropathic pain & 53 & 3.67 & $6,277.49$ & 118.44 & 95.02 \\
\hline Proteolin & Joint disorder pain/inflammation & 52 & 3.60 & $8,086.08$ & 155.50 & 94.52 \\
\hline Sentra PM & Insomnia & 42 & 2.90 & $7,648.45$ & 182.11 & 130.21 \\
\hline Deplin & Major depression & 37 & 2.56 & $4,188.02$ & 113.19 & 19.40 \\
\hline Sentra AM & Insomnia & 33 & 2.28 & $8,856.49$ & 268.38 & 323.02 \\
\hline Folbic & Atherosclerosis & 22 & 1.52 & 503.15 & 22.87 & 10.48 \\
\hline Trepadone & Joint disorder pain/inflammation & 19 & 1.31 & $4,948.09$ & 260.43 & 274.67 \\
\hline GABAdone & Insomnia & 18 & 1.24 & $2,569.77$ & 142.77 & 149.74 \\
\hline Apptrim & Obesity & 16 & 1.11 & $6,302.57$ & 393.91 & 261.53 \\
\hline Foltanx & Diabetic neuropathy & 14 & 0.97 & $1,099.63$ & 78.55 & 0.45 \\
\hline Cerefolin & Cognitive impairment, hyperhomocysteinemia & 13 & 0.90 & $1,210.01$ & 93.08 & 86.59 \\
\hline FABB & Nutrition in cystic fibrosis & 10 & 0.69 & 246.60 & 24.66 & 6.75 \\
\hline VSL\#3 & Ulcerative colitis & 3 & 0.21 & 487.87 & 162.62 & 56.93 \\
\hline Ferrex & Iron deficiency, anemia & 1 & 0.07 & 102.16 & 102.16 & - \\
\hline Hypertensa & Hypertension & 1 & 0.07 & 0.00 & 0.00 & - \\
\hline \multicolumn{2}{|l|}{ Total } & 1,446 & 100.00 & $219,889.00$ & 152.00 & 203.86 \\
\hline
\end{tabular}

${ }^{a}$ Name of medical food indicated on claims.

bIndication of medical food.

cNumber of claims reimbursed.

${ }^{d}$ Percentage out of all reimbursed medical food during the time frame.

'Total amount reimbursed for the medical food during the time frame in U.S. dollars.

$f_{M e a n}$ amount reimbursed for the medical food during the time frame in U.S. dollars.

sStandard deviation from the mean amount reimbursed for the medical food during the time frame. 
difference in mean amount reimbursed per claim for pharmacydispensed MFs and CPs before and after the implementation of $A B$ 378; it was hypothesized that there should be no statistically significant difference, as AB 378 did not regulate pharmacy-dispensed MFs and CPs.

\section{Results}

The CAWCS database contained a total of 20,373,477 claims during the study period of January 1, 2011, through December 31, 2013. Of these, 4,641 claims were for dispensing fees and $7,838,859$ claims were for medical devices and supplies. The remaining 12,529,977 claims were for pharmaceutical products. MFs and CPs represented approximately 151,000 out of 20 million total claims (each representing a prescription) dispensed in CAWCS across all 3 years of the study, or about 51,000 prescriptions annually.

Of all MF and CP prescriptions, 95\% were physician-dispensed (5\% were pharmacy-dispensed). Out of 151,107 MFs and CPs dispensed by both physicians and pharmacies, 95,528 (63.2\%) prescriptions were reimbursed. Of the 95,528 reimbursed prescriptions, 76,056 were MF prescriptions and 19,482 were CP prescriptions. Physicians dispensed 54 different MFs and CPs, and pharmacies dispensed 46 different MFs and CPs. The reimbursed MFs and CPs accounted for approximately $\$ 19$ million paid over the 3 years. Of the total amount paid by CAWCS, $\$ 18.5$ million (96.4\%) was paid to physicians and $\$ 682,000$ (3.6\%) was paid to pharmacies.

Table 1 contains the number of prescriptions and per prescription cost of MFs reimbursed by CAWCS by named MF and by dispenser (physician or pharmacy) from 2011 to 2013. The most prescribed MF by physicians was Theramine for chronic low back pain and by pharmacies was Somnicin for insomnia. The top $4 \mathrm{MFs}$ for both dispensers were all for either insomnia or inflammatory pain relief. Physicians' most frequently reimbursed MF was Theramine (48.5\%), followed by Sentra PM (17.7\%), Sentra AM (10.3\%), and GABAdone (7.9\%), all for insomnia. The fifth and sixth most reimbursed MFs were for joint inflammatory pain: Trepadone (4.0\%) and Proteolin (3.7\%). Following MFs for pain were Somnicin (2.8\%) for insomnia, Apptrim (2.5\%) for obesity, and others (2.7\%) for indications such as osteoarthritis, hypertension, and depression. Theramine alone accounted for $\$ 8.5$ million spent, which was more than half of the total amount reimbursed for physician-dispensed MFs. Theramine also had the second highest reimbursed amount per prescription (\$244), slightly under Trepadone (\$270), which also was used for chronic inflammatory pain.

The most frequently reimbursed pharmacy-dispensed MFs was Somnicin $(25.0 \%)$ for insomnia, followed by Theramine (17.3\%) for chronic low back pain, Limbrel (9.2\%) and Proteolin (7.7\%) for joint inflammatory pain, Deplin (5.8\%) for major depression, L-methylfolate (5.0\%) for renal impairment, and Sentra PM (4.9\%), GABAdone (4.8\%), and Sintralyne-PM (4.8\%), all for insomnia. While Somnicin was the most frequently reimbursed pharmacy-dispensed MF, Theramine was the costliest MF, costing CAWCS $\$ 225,143$ and $\$ 325$ per prescription.

The top reimbursed MFs did not vary greatly by year (Table 1 ). The only exception was Somnicin, which became the top reimbursed medication in 2012 immediately following its availability on the market.

Table 2 contains the number of $\mathrm{CP}$ prescriptions and mean amount reimbursed per prescription by CPs and by dispenser between 2011 and 2013. The most frequently reimbursed physician-dispensed CP was Theraproxen (11.8\%) for the indications of inflammation and pain, followed by Gaboxetine (11.7\%) for major depressive disorder, Theratramadol (10.7\%) for pain, Theraprofen (9.7\%) for inflammation, and Prazolamine (9.2\%) for painful musculoskeletal conditions. Therapoxyphene for pain, with only 5 reimbursed prescriptions, had the highest reimbursed amount per prescription for physician-dispensed CP (\$423.30), and Sentraflox AM-10 for major depressive disorder had the second highest (\$393.79).

The most frequently reimbursed pharmacy-dispensed CP over the 3 years was Theraprofen (16.1\%) for inflammation, followed by Theracodophen (13.0\%), Theraproxen (10.31\%), and Theratramadol (10.31\%), which were all for pain. Sentraflox AM-10 had the highest reimbursed amount per prescription (\$811.77) for pharmacy-dispensed CPs. Trepoxen, with only 2 reimbursed prescriptions, had the second highest reimbursed amount per prescription (\$625.95) for pharmacy-dispensed CPs.

While the top reimbursed CPs varied slightly over the years (Table 2), for both physician-dispensed and pharmacydispensed CPs, the top medications were for pain management.

A steady reduction was observed in both the yearly number of physician-dispensed MFs (from 54,325 in 2011 to 31,998 in 2012 to 27,201 in 2013), and CPs billed (from 17,605 in 2011 to 8,874 in 2012 to 3,540 in 2013) and the number of reimbursed (paid) physician-dispensed MFs (from 34,192 in 2011 to 20,486 in 2012 to 17,376 in 2013) and CPs (from 11,162 in 2011 to 5,900 in 2012 to 2,197 in 2013). The percentage of physician-dispensed billed prescriptions that were reimbursed remained steady from $62.9 \%$ in 2011 to $64.0 \%$ in 2012 to $63.9 \%$ in 2013. This trend may be indicative that CAWCS did not change its reimbursement pattern, but physicians were simply prescribing and billing fewer MFs (Table 3).

Pharmacy-dispensed MFs and CPs, which were not regulated by $\mathrm{AB} 378$, did not experience a reduction but did experience fluctuation in prescriptions and reimbursements. The yearly number of pharmacy-dispensed MFs billed increased from 1,698 in 2011 to 2,357 in 2012 to 3,056 in 2013, with increased numbers reimbursed from 1,129 in 2011 to 1,427 in 2012 to 1,446 in 2013, while the percentage of the prescriptions that were reimbursed decreased from $66.5 \%$ in 2011 to $60.5 \%$ in 2012 to $47.3 \%$ in 2013. 
TABLE 2 Convenience Packages by Dispenser from 2011 to 2013

Co-pack ${ }^{\mathrm{a}}$

Indication $^{b}$

Prescriptions

Reimbursedc $^{c}$ Percentaged $^{\mathrm{d}}$

Total Amount Mean Amount

Reimbursed, Reimbursed,

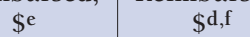

Standard

Deviationg

Dispensed by Physicians

2011-2013

\begin{tabular}{|c|}
\hline Theraproxen \\
\hline Gaboxetine \\
\hline Theratramadol \\
\hline Theraprofen \\
\hline Prazolamine \\
\hline Gabitidine \\
\hline Therabenzaprine \\
\hline Gabazolamine \\
\hline Theracodophen \\
\hline Strazepam \\
\hline Therapentin \\
\hline Lytensopril \\
\hline Appformin \\
\hline Hypertensolol \\
\hline Trepoxicam \\
\hline Sentraflox AM-10 \\
\hline Sentrazolpidem \\
\hline Sentradine \\
\hline Trepoxen \\
\hline Theracodeine \\
\hline Trazamine \\
\hline Gabazolpidem-5 \\
\hline Therafeldamine \\
\hline Hypertenipine-2.5 \\
\hline Therapoxyphene \\
\hline Total \\
\hline 2011 \\
\hline
\end{tabular}

Inflammation and pain

Major depressive disorder

Moderate to severe pain

Inflammation

Discomfort associated with acute, painful musculoskeletal conditions

Active duodenal ulcer

Muscle spasm, inflammation, pain disorder

Anxiety disorder

Moderate to moderately severe pain

Insomnia

Postherpetic neuralgia, partial seizure

Hypertension

Type II diabetes

Hypertension

Osteoarthritis of the knee and hip

Major depressive disorder, OCD

Insomnia

Active duodenal ulcer

Pain disorders, inflammation

Pain disorders, inflammation

Sleep disorders

Insomnia

Osteoarthritis, rheumatoid arthritis

Hypertension

Mild to moderate pain

Prazolamine

Theraproxen

Gaboxetine

Gabazolamine

Theraprofen

Theratramadol

Gabitidine

Therabenzaprine

Theracodophen

Strazepam

Therapentin

Lytensopril

Hypertensolol

Sentradine

Appformin

Theracodeine

Trepoxicam

Sentraflox AM-10

Sentrazolpidem

Trazamine

Trepoxen

Therafeldamine

\begin{tabular}{|l|r}
\hline $\begin{array}{l}\text { Discomfort associated with acute, painful } \\
\text { musculoskeletal conditions }\end{array}$ & 1,392 \\
\hline Inflammation and pain & 1,360 \\
\hline Major depressive disorder & 1,309 \\
\hline Anxiety disorder & 1,288 \\
\hline Inflammation & 1,237 \\
\hline Moderate to severe pain & 985 \\
\hline Active duodenal ulcer & 826 \\
\hline Muscle spasm, inflammation, pain disorder & 705 \\
\hline Moderate to moderately severe pain & 332 \\
\hline Insomnia & 261 \\
\hline Postherpetic neuralgia, partial seizure & 177 \\
\hline Hypertension & 127 \\
\hline Hypertension & 71 \\
\hline Active duodenal ulcer & 40 \\
\hline Type II diabetes & 34 \\
\hline Pain disorders, inflammation & 26 \\
\hline Osteoarthritis of the knee and hip & 24 \\
\hline Major depressive disorder, OCD & 14 \\
\hline Insomnia & \\
\hline Sleep disorders & \\
\hline Pain disorders, inflammation & \\
\hline Osteoarthritis, rheumatoid arthritis & \\
\hline
\end{tabular}

2,273

2,252

2,061

1,870

11.80

11.80

10.70

1,767

9.71

9.17

\begin{tabular}{l|l}
1,680 & 8.72
\end{tabular}

8.72

1,644

1,481

1,217

$\begin{array}{r}8.54 \\ 7.69 \\ \hline\end{array}$

1

\begin{tabular}{|l|}
\hline $518,198.11$ \\
\hline $641,056.48$ \\
\hline $641,733.24$ \\
\hline $437,055.93$ \\
\hline $328,296.51$ \\
\hline
\end{tabular}

$464,939.91$

$385,324.99$

6.32

$305,588.41$

\begin{tabular}{l|l|l}
\hline 810 & 4.21 & $128,730.24$ \\
\hline 650 & 3.38 & $238,211.88$
\end{tabular}

$238,211.88$

\begin{tabular}{l|l}
650 & 3.38 \\
\hline 365 & 1.90
\end{tabular}

1.90

$238,211.88$

\begin{tabular}{l|l|l}
3228 & 1.18 & $48,241.4$ \\
\hline
\end{tabular}

$43,584.99$

\begin{tabular}{l|l}
175 & 0.91 \\
151 & 0.78 \\
\hline
\end{tabular}

\begin{tabular}{l|l}
151 & 0.78 \\
\hline 136 & 0.71
\end{tabular}

$44,506.97$

\begin{tabular}{l|l|l}
130 & 0.68 & $31,625.94$ \\
\hline
\end{tabular}

$23,231.33$

110

110
70

\begin{tabular}{|l}
\hline \\
\hline \\
\hline
\end{tabular}

0.57

0.36

0.33

0.25

$16,621.80$

0.23

$12,592.30$

0.11

0.03

0.03

\begin{tabular}{|r|}
\hline $6,547.96$ \\
\hline $4,246.33$ \\
\hline 511.80 \\
\hline $2,116.51$ \\
\hline $4,747,418.27$ \\
\hline
\end{tabular}

\begin{tabular}{|c|c|c|}
\hline 227.98 & 195.62 \\
\hline & 284.66 & 373.94 \\
\hline & 311.37 & 306.50 \\
\hline & 233.72 & 179.39 \\
\hline & 185.79 & 154.30 \\
\hline
\end{tabular}

276.75

303.70

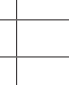

234.38

207.15

205.12

251.10

218.98

158.93

366.48

145.33

211.59

249.06

294.75

393.79

243.28

211.19

237.45

172.93

256.99

148.82

193.02

102.36

423.30

198.12

117.00

420.14

237.16

374.41

361.67

241.38

376.50

268.52

239.51

351.19

166.07

259.27

220.59

151.71

135.16

19,259

100.00

246.50

258.23

\begin{tabular}{r|r|r|r|r}
1,392 & 12.47 & $245,346.77$ & 176.25 & 143.29 \\
\hline 1,360 & 12.18 & $271,581.71$ & 199.69 & 174.82 \\
\hline 1,309 & 11.73 & $462,521.02$ & 353.34 & 434.22 \\
\hline 1,288 & 11.54 & $276,142.03$ & 214.40 & 223.68 \\
\hline 1,237 & 11.08 & $275,696.74$ & 222.88 & 159.89 \\
\hline 985 & 8.82 & $299,860.96$ & 304.43 & 297.65 \\
\hline 826 & 7.40 & $212,021.71$ & 256.68 & 299.19 \\
\hline 805 & 7.21 & $205,278.26$ & 255.00 & 279.60 \\
\hline 701 & 6.28 & $177,649.43$ & 253.42 & 213.93 \\
\hline 332 & 2.97 & $54,374.14$ & 163.78 & 118.02 \\
\hline 261 & 2.34 & $97,192.74$ & 372.39 & 363.71 \\
\hline 177 & 1.59 & $25,124.07$ & 141.94 & 243.53 \\
\hline 127 & 1.14 & $20,626.58$ & 162.41 & 270.51 \\
\hline 91 & 0.82 & $18,509.53$ & 203.40 & 230.38 \\
\hline 71 & 0.64 & $15,346.76$ & 216.15 & 399.17 \\
\hline 40 & 0.36 & $5,167.23$ & 129.18 & 160.25 \\
\hline 34 & 0.30 & $10,473.38$ & 308.04 & 223.57 \\
\hline 26 & 0.23 & $10,632.20$ & 408.93 & 411.72 \\
\hline 25 & 0.22 & $6,639.24$ & 265.57 & 271.78 \\
\hline 24 & 0.22 & $7,306.39$ & 304.43 & 316.35 \\
\hline 19 & 0.17 & $7,260.99$ & 382.16 & 373.35 \\
\hline 14 & 0.13 & $1,769.65$ & 126.40 & 154.71 \\
\hline & & & & \\
\hline
\end{tabular}

continued on next page 
TABLE 2 Convenience Packages by Dispenser from 2011 to 2013 (continued)

\begin{tabular}{|c|c|c|c|c|c|c|}
\hline Co-pack ${ }^{a}$ & Indication $^{b}$ & $\begin{array}{l}\text { Prescriptions } \\
\text { Reimbursed }\end{array}$ & Percentage $^{d}$ & $\begin{array}{c}\text { Total Amount } \\
\text { Reimbursed, } \\
\text { \$e }\end{array}$ & $\begin{array}{c}\text { Mean Amount } \\
\text { Reimbursed, } \\
\$ \text { d,f }\end{array}$ & $\begin{array}{l}\text { Standard } \\
\text { Deviationg }\end{array}$ \\
\hline \multicolumn{7}{|l|}{2011} \\
\hline Gabazolpidem-5 & Insomnia & 10 & 0.09 & $1,832.20$ & 183.22 & 208.51 \\
\hline Hypertenipine- 2.5 & Hypertension & 4 & 0.04 & 500.68 & 125.17 & 144.53 \\
\hline Therapoxyphene & Mild to moderate pain & 4 & 0.04 & $2,116.51$ & 529.13 & 119.39 \\
\hline \multicolumn{2}{|l|}{ Total } & 11,162 & 100.00 & $2,710,970.92$ & 242.88 & 267.65 \\
\hline \multicolumn{7}{|l|}{2012} \\
\hline Theraproxen & Inflammation and pain & 771 & 13.07 & $197,498.24$ & 256.16 & 201.33 \\
\hline Therabenzaprine & Muscle spasm, inflammation, pain disorder & 739 & 12.53 & $150,026.67$ & 203.01 & 198.19 \\
\hline Theratramadol & Moderate to severe pain & 694 & 11.76 & $212,840.24$ & 306.69 & 312.45 \\
\hline Gabitidine & Active duodenal ulcer & 646 & 10.95 & $177,184.39$ & 274.28 & 302.25 \\
\hline Gaboxetine & Major depressive disorder & 603 & 10.22 & $116,814.37$ & 193.72 & 260.38 \\
\hline Theraprofen & Inflammation & 502 & 8.51 & $121,472.94$ & 241.98 & 193.87 \\
\hline Theracodophen & Moderate to moderately severe pain & 448 & 7.59 & $113,562.36$ & 253.49 & 179.91 \\
\hline Prazolamine & $\begin{array}{l}\text { Discomfort associated with acute, painful } \\
\text { musculoskeletal conditions }\end{array}$ & 292 & 4.95 & $58,749.31$ & 201.20 & 177.40 \\
\hline Strazepam & Insomnia & 243 & 4.12 & $38,454.08$ & 158.25 & 127.35 \\
\hline Therapentin & Postherpetic neuralgia, partial seizure & 238 & 4.03 & $85,102.39$ & 357.57 & 503.88 \\
\hline Gabazolamine & Anxiety disorder & 192 & 3.25 & $30,498.40$ & 158.85 & 178.06 \\
\hline Lytensopril & Hypertension & 130 & 2.20 & $17,546.06$ & 134.97 & 221.68 \\
\hline Appformin & Type II diabetes & 94 & 1.59 & $16,523.50$ & 175.78 & 348.60 \\
\hline Trepoxicam & Osteoarthritis of the knee and hip & 64 & 1.08 & $18,324.87$ & 286.33 & 264.85 \\
\hline Sentraflox AM-10 & Major depressive disorder, OCD & 54 & 0.92 & $22,131.30$ & 409.84 & 382.79 \\
\hline Sentrazolpidem & Insomnia & 45 & 0.76 & $12,097.35$ & 268.83 & 323.15 \\
\hline Hypertensolol & Hypertension & 38 & 0.64 & $14,483.74$ & 381.15 & 421.68 \\
\hline Trepoxen & Pain disorders, inflammation & 26 & 0.44 & $3,951.30$ & 151.97 & 298.20 \\
\hline Gabazolpidem-5 & Insomnia & 25 & 0.42 & $4,002.73$ & 160.11 & 250.77 \\
\hline Theracodeine & Pain disorders, inflammation & 24 & 0.41 & $5,899.98$ & 245.83 & 152.06 \\
\hline Trazamine & Sleep disorders & 23 & 0.39 & $5,269.55$ & 229.11 & 182.03 \\
\hline Sentradine & Active duodenal ulcer & 5 & 0.08 & 670.42 & 134.08 & 183.60 \\
\hline Therafeldamine & Osteoarthritis, rheumatoid arthritis & 2 & 0.03 & 664.14 & 332.07 & 0.00 \\
\hline Hypertenipine-2.5 & Hypertension & 1 & 0.02 & 11.12 & 11.12 & - \\
\hline Therapoxyphene & Mild to moderate pain & 1 & 0.02 & 0.00 & 0.00 & - \\
\hline \multicolumn{2}{|l|}{ Total } & 5,900 & 100.00 & $1,423,779.45$ & 241.32 & 261.77 \\
\hline \multicolumn{7}{|l|}{2013} \\
\hline Theratramadol & Moderate to severe pain & 382 & 17.39 & $129,032.04$ & 337.78 & 317.31 \\
\hline Gaboxetine & Major depressive disorder & 340 & 15.48 & $61,721.09$ & 181.53 & 192.40 \\
\hline Strazepam & Insomnia & 235 & 10.70 & $35,902.02$ & 152.77 & 103.81 \\
\hline Gabitidine & Active duodenal ulcer & 208 & 9.47 & $75,733.81$ & 364.10 & 312.11 \\
\hline Therapentin & Postherpetic neuralgia, partial seizure & 151 & 6.87 & $55,916.75$ & 370.31 & 364.80 \\
\hline Theraproxen & Inflammation and pain & 142 & 6.46 & $49,118.16$ & 345.90 & 273.21 \\
\hline Theraprofen & Inflammation & 131 & 5.96 & $39,886.25$ & 304.48 & 261.32 \\
\hline Therabenzaprine & Muscle spasm, inflammation, pain disorder & 100 & 4.55 & $30,020.06$ & 300.20 & 308.72 \\
\hline Prazolamine & $\begin{array}{l}\text { Discomfort associated with acute, painful } \\
\text { musculoskeletal conditions }\end{array}$ & 83 & 3.78 & $24,200.43$ & 291.57 & 198.43 \\
\hline Theracodophen & Moderate to moderately severe pain & 68 & 3.10 & $14,376.62$ & 211.42 & 128.68 \\
\hline Appformin & Type II diabetes & 63 & 2.87 & $16,371.15$ & 259.86 & 383.06 \\
\hline Sentrazolpidem & Insomnia & 60 & 2.73 & $12,889.35$ & 214.82 & 219.54 \\
\hline Lytensopril & Hypertension & 58 & 2.64 & $10,375.22$ & 178.88 & 251.83 \\
\hline Sentraflox AM-10 & Major depressive disorder, OCD & 56 & 2.55 & $20,791.47$ & 371.28 & 358.89 \\
\hline Trepoxicam & Osteoarthritis of the knee and hip & 53 & 2.41 & $15,708.72$ & 296.39 & 226.20 \\
\hline Trepoxen & Pain disorders, inflammation & 25 & 1.14 & $5,409.51$ & 216.38 & 363.94 \\
\hline Sentradine & Active duodenal ulcer & 14 & 0.64 & $4,051.38$ & 289.38 & 306.37 \\
\hline Hypertensolol & Hypertension & 10 & 0.46 & $8,474.67$ & 847.47 & 442.63 \\
\hline
\end{tabular}


TABLE 2 Convenience Packages by Dispenser from 2011 to 2013 (continued)

\begin{tabular}{|c|c|c|c|c|c|c|}
\hline Co-pack ${ }^{a}$ & Indication ${ }^{b}$ & $\begin{array}{l}\text { Prescriptions } \\
\text { Reimbursed }\end{array}$ & Percentaged $^{d}$ & $\begin{array}{c}\text { Total Amount } \\
\text { Reimbursed, } \\
\text { \$e }\end{array}$ & $\begin{array}{c}\text { Mean Amount } \\
\text { Reimbursed, } \\
\$ \mathrm{~d}, \mathrm{f}\end{array}$ & $\begin{array}{l}\text { Standard } \\
\text { Deviationg }\end{array}$ \\
\hline \multicolumn{7}{|l|}{2013} \\
\hline Gabazolpidem-5 & Insomnia & 9 & 0.41 & 713.03 & 79.23 & 129.00 \\
\hline Therafeldamine & Osteoarthritis, rheumatoid arthritis & 6 & 0.27 & $1,812.54$ & 302.09 & 0.00 \\
\hline Trazamine & Sleep disorders & 2 & 0.09 & 16.36 & 8.18 & 0.00 \\
\hline Gabazolamine & Anxiety disorder & 1 & 0.05 & 147.27 & 147.27 & - \\
\hline \multicolumn{2}{|l|}{ Total } & 2,197 & 100.00 & $612,667.90$ & 278.87 & 282.85 \\
\hline \multicolumn{7}{|c|}{ Dispensed by Pharmacies } \\
\hline \multicolumn{7}{|c|}{$2011-2013$} \\
\hline Theraprofen & Inflammation & 36 & 16.14 & $9,342.92$ & 259.53 & 225.93 \\
\hline Theracodophen & Moderate to moderately severe pain & 29 & 13.00 & $6,522.39$ & 224.91 & 213.28 \\
\hline Theraproxen & Inflammation and pain & 23 & 10.31 & $6,737.76$ & 292.95 & 180.41 \\
\hline Theratramadol & Moderate to severe pain & 23 & 10.31 & $5,952.43$ & 258.80 & 335.60 \\
\hline Gabitidine & Active duodenal ulcer & 19 & 8.52 & $5,799.63$ & 305.24 & 204.34 \\
\hline Therabenzaprine & Muscle spasm, inflammation, pain disorder & 16 & 7.17 & $1,709.14$ & 106.82 & 161.29 \\
\hline Strazepam & Insomnia & 12 & 5.38 & $2,133.05$ & 177.75 & 125.78 \\
\hline Trepoxicam & Osteoarthritis of the knee and hip & 11 & 4.93 & $3,498.23$ & 318.02 & 139.03 \\
\hline Sentraflox AM-10 & Major depressive disorder, OCD & 6 & 2.69 & $4,870.60$ & 811.77 & 151.59 \\
\hline Theracodeine & Pain disorders and inflammation & 6 & 2.69 & $1,901.13$ & 316.86 & 8.62 \\
\hline Appformin & Type II diabetes & 5 & 2.24 & $1,118.45$ & 223.69 & 467.91 \\
\hline Gabazolpidem-5 & Insomnia & 5 & 2.24 & 978.99 & 195.80 & 185.26 \\
\hline Gaboxetine & Major depressive disorder & 5 & 2.24 & $1,968.31$ & 393.66 & 295.05 \\
\hline Gabazolamine & Anxiety disorder & 4 & 1.79 & $1,706.14$ & 426.54 & 92.55 \\
\hline Lytensopril & Hypertension & 4 & 1.79 & $1,174.20$ & 293.55 & 0.00 \\
\hline Prazolamine & $\begin{array}{l}\text { Discomfort associated with acute, painful } \\
\text { musculoskeletal conditions }\end{array}$ & 4 & 1.79 & 0.00 & 0.00 & 0.00 \\
\hline Sentradine & Active duodenal ulcer & 4 & 1.79 & 0.00 & 0.00 & 0.00 \\
\hline Sentrazolpidem & Insomnia & 3 & 1.35 & $1,573.25$ & 524.42 & 505.81 \\
\hline Therapentin & Postherpetic neuralgia, partial seizure & 3 & 1.35 & $1,214.14$ & 404.71 & 658.53 \\
\hline Trazamine & Sleep disorders & 2 & 0.90 & 883.24 & 441.62 & 204.76 \\
\hline Trepoxen & Pain disorders, inflammation & 2 & 0.90 & $1,251.90$ & 625.95 & 0.00 \\
\hline Therafeldamine & Osteoarthritis, rheumatoid arthritis & 1 & 0.45 & 335.66 & 335.66 & - \\
\hline \multicolumn{2}{|l|}{ Total } & 223 & 100.00 & $60,671.56$ & 272.07 & 255.38 \\
\hline \multicolumn{7}{|l|}{2011} \\
\hline Theracodophen & Moderate to moderately severe pain & 13 & 16.67 & $3,185.69$ & 245.05 & 263.15 \\
\hline Theratramadol & Moderate to severe pain & 9 & 11.54 & $2,263.08$ & 251.45 & 334.26 \\
\hline Strazepam & Insomnia & 7 & 8.97 & $1,079.61$ & 154.23 & 139.03 \\
\hline Theraprofen & Inflammation & 6 & 7.69 & $1,774.39$ & 295.73 & 254.82 \\
\hline Theracodeine & Pain disorders and inflammation & 5 & 6.41 & $1,566.86$ & 313.37 & 1.41 \\
\hline Trepoxicam & Osteoarthritis of the knee and hip & 5 & 6.41 & $1,543.32$ & 308.66 & 216.57 \\
\hline Gabazolamine & Anxiety disorder & 4 & 5.13 & $1,706.14$ & 426.54 & 92.55 \\
\hline Prazolamine & $\begin{array}{l}\text { Discomfort associated with acute, painful } \\
\text { musculoskeletal conditions }\end{array}$ & 4 & 5.13 & 0.00 & 0.00 & 0.00 \\
\hline Theraproxen & Inflammation and pain & 4 & 5.13 & $1,512.64$ & 378.16 & 35.51 \\
\hline Gabitidine & Active duodenal ulcer & 3 & 3.85 & $1,709.93$ & 569.98 & 194.93 \\
\hline Gaboxetine & Major depressive disorder & 3 & 3.85 & $1,760.16$ & 586.72 & 153.34 \\
\hline Lytensopril & Hypertension & 3 & 3.85 & 880.65 & 293.55 & 0.00 \\
\hline Therabenzaprine & Muscle spasm, inflammation, pain disorder & 3 & 3.85 & 339.96 & 113.32 & 196.28 \\
\hline Gabazolpidem-5 & Insomnia & 2 & 2.56 & 735.43 & 367.72 & 169.92 \\
\hline Sentrazolpidem & Insomnia & 2 & 2.56 & 563.95 & 281.98 & 398.77 \\
\hline Trazamine & Sleep disorders & 2 & 2.56 & 883.24 & 441.62 & 204.76 \\
\hline Trepoxen & Pain disorders, inflammation & 2 & 2.56 & $1,251.90$ & 625.95 & 0.00 \\
\hline Therafeldamine & Osteoarthritis, rheumatoid arthritis & 1 & 1.28 & 335.66 & 335.66 & - \\
\hline \multicolumn{2}{|l|}{ Total } & 78 & 100.00 & $23,092.61$ & 296.06 & 236.42 \\
\hline
\end{tabular}


TABLE 2 Convenience Packages by Dispenser from 2011 to 2013 (continued)

\begin{tabular}{|c|c|c|c|c|c|c|}
\hline Co-pack ${ }^{a}$ & Indication $^{\mathrm{b}}$ & $\begin{array}{l}\text { Prescriptions } \\
\text { Reimbursed }^{c}\end{array}$ & Percentaged $^{d}$ & $\begin{array}{c}\text { Total Amount } \\
\text { Reimbursed, } \\
\$ \mathrm{Se}\end{array}$ & $\begin{array}{c}\text { Mean Amount } \\
\text { Reimbursed, } \\
\$_{\mathrm{d}, \mathrm{f}}\end{array}$ & $\begin{array}{c}\text { Standard } \\
\text { Deviationg }\end{array}$ \\
\hline \multicolumn{7}{|l|}{2012} \\
\hline Theraprofen & Inflammation & 24 & 21.43 & $4,927.15$ & 205.30 & 166.54 \\
\hline Theraproxen & Inflammation and pain & 19 & 16.96 & $5,225.12$ & 275.01 & 193.95 \\
\hline Gabitidine & Active duodenal ulcer & 14 & 12.50 & $3,996.11$ & 285.44 & 158.42 \\
\hline Therabenzaprine & Muscle spasm, inflammation, pain disorder & 13 & 11.61 & $1,369.18$ & 105.32 & 161.50 \\
\hline Theracodophen & Moderate to moderately severe pain & 13 & 11.61 & $3,303.07$ & 254.08 & 155.28 \\
\hline Theratramadol & Moderate to severe pain & 8 & 7.14 & $3,598.86$ & 449.86 & 365.44 \\
\hline Sentraflox AM-10 & Major depressive disorder, OCD & 5 & 4.46 & $3,749.40$ & 749.88 & 0.00 \\
\hline Trepoxicam & Osteoarthritis of the knee and hip & 5 & 4.46 & $1,629.93$ & 325.99 & 34.96 \\
\hline Sentradine & Active duodenal ulcer & 4 & 3.57 & 0.00 & 0.00 & 0.00 \\
\hline Appformin & Type II diabetes & 3 & 2.68 & 33.03 & 11.01 & 0.00 \\
\hline Gabazolpidem-5 & Insomnia & 1 & 0.89 & 0.00 & 0.00 & - \\
\hline Gaboxetine & Major depressive disorder & 1 & 0.89 & 208.15 & 208.15 & - \\
\hline Lytensopril & Hypertension & 1 & 0.89 & 293.55 & 293.55 & - \\
\hline Theracodeine & Pain disorders and inflammation & 1 & 0.89 & 334.27 & 334.27 & - \\
\hline \multicolumn{2}{|l|}{ Total } & 112 & 100.00 & $28,667.82$ & 255.96 & 225.04 \\
\hline \multicolumn{7}{|l|}{2013} \\
\hline Theraprofen & Inflammation & 6 & 18.18 & $2,641.38$ & 440.23 & 331.55 \\
\hline Theratramadol & Moderate to severe pain & 6 & 18.18 & 90.49 & 15.08 & 7.87 \\
\hline Strazepam & Insomnia & 5 & 15.15 & $1,053.44$ & 210.69 & 110.40 \\
\hline Theracodophen & Moderate to moderately severe pain & 3 & 9.09 & 33.63 & 11.21 & 0.00 \\
\hline Therapentin & Postherpetic neuralgia, partial seizure & 3 & 9.09 & $1,214.14$ & 404.71 & 658.53 \\
\hline Appformin & Type II diabetes & 2 & 6.06 & $1,085.42$ & 542.71 & 732.48 \\
\hline Gabazolpidem-5 & Insomnia & 2 & 6.06 & 243.56 & 121.78 & 0.00 \\
\hline Gabitidine & Active duodenal ulcer & 2 & 6.06 & 93.59 & 46.80 & 0.01 \\
\hline Gaboxetine & Major depressive disorder & 1 & 3.03 & 0.00 & 0.00 & - \\
\hline Sentraflox AM-10 & Major depressive disorder, OCD & 1 & 3.03 & $1,121.20$ & $1,121.20$ & - \\
\hline Sentrazolpidem & Insomnia & 1 & 3.03 & $1,009.30$ & $1,009.30$ & - \\
\hline Trepoxicam & Osteoarthritis of the knee and hip & 1 & 3.03 & 324.98 & 324.98 & - \\
\hline \multicolumn{2}{|l|}{ Total } & 33 & 100.00 & $8,911.13$ & 270.03 & 374.11 \\
\hline
\end{tabular}

aName of convenience package indicated on claims.

IIndication of convenience package.

cNumber of claims reimbursed.

dPercentage out of all reimbursed convenience packages during the time frame.

'Total amount reimbursed for the convenience package during the time frame in U.S. dollars.

${ }^{f}$ Mean amount reimbursed for the convenience package during the time frame in U.S. dollars.

sStandard deviation from the mean amount reimbursed for the convenience package during the time frame.

Co-pack = convenience package; $O C D=$ obsessive compulsive disorder.

Pharmacy-dispensed CPs billed versus those reimbursed experienced an interesting pattern between 2011 and 2013, with the CP prescriptions billed decreasing steadily from 225 in 2011 to 175 in 2012 to 53 in 2013, but the reimbursed number fluctuated from 78 prescriptions in 2011 to 112 in 2012 to 22 in 2013. CAWCS reimbursed a significantly higher percentage of billed CPs in 2012 (64.0\%) than in 2011 (34.7\%) and in 2013 (62.3\%). The pattern signaled a spillover effect in which pharmacies not subject to the regulations of $A B 378$ were dispensing more MFs because physicians were facing these AB 378 regulations and dispensing fewer MFs.
The amount paid by CAWCS for MFs and CPs was examined to gain a deeper understanding of the changes in physician behavior and the potential spillover effects in response to differential AB 378, which, starting in 2012, applied only to physicians. CAWCS paid $\$ 18,466,990$ for physician-dispensed MFs and CPs; this amount accounted for $96 \%$ of MF and CP total cost in CAWCS between 2011 and 2013 (Table 3). Pharmacydispensed MFs and CPs accounted for the remaining $\$ 0.7$ million (4\% of total cost). The mean amount reimbursed per prescription across the 3 years for MFs was higher when physiciandispensed ( $\$ 190.41$, standard deviation $[S D]=203.97$ ) than when pharmacy-dispensed $(\$ 155.24, \mathrm{SD}=200.72)$. Conversely, 
TABLE 3 Billed and Reimbursed Medical Foods and Convenience Packages by Dispenser from 2011 to 2013

\begin{tabular}{|c|c|c|c|c|c|c|c|c|}
\hline Billed, Year & $\begin{array}{l}\text { Prescriptions } \\
\text { Billed }^{\mathrm{a}}\end{array}$ & $\begin{array}{c}\text { Reimbursed, } \\
\text { Year }\end{array}$ & $\begin{array}{l}\text { Prescriptions } \\
\text { Reimbursed }^{\text {b }}\end{array}$ & $\begin{array}{c}\text { Percentage } \\
\text { Reimbursed }\end{array}$ & Median, $\$ \$^{\mathrm{d}}$ & Mean, \$e & $\begin{array}{l}\text { Standard } \\
\text { Deviation }\end{array}$ & $\begin{array}{c}\text { Total } \\
\text { Reimbursed } \\
\text { Amount, } \$ g\end{array}$ \\
\hline \multicolumn{9}{|c|}{ Dispensed by Physicians } \\
\hline $\begin{array}{l}\text { MF } \\
(2011-2013)\end{array}$ & 113,524 & $\begin{array}{c}\text { MF } \\
\text { (2011-2013) }\end{array}$ & 72,054 & 63.5 & 188.56 & 190.41 & 203.97 & $13,719,572$ \\
\hline 2011 & 54,325 & 2011 & 34,192 & 62.9 & 194.51 & 195.64 & 206.96 & $6,689,187$ \\
\hline 2012 & 31,998 & 2012 & 20,486 & 64.0 & 190.24 & 196.21 & 197.11 & $4,019,602$ \\
\hline 2013 & 27,201 & 2013 & 17,376 & 63.9 & 172.22 & 173.27 & 205.05 & $3,010,783$ \\
\hline $\begin{array}{l}\text { CP } \\
(2011-2013)\end{array}$ & 30,019 & $\begin{array}{c}C P \\
(2011-2013) \\
\end{array}$ & 19,259 & 64.2 & 221.05 & 246.50 & 267.89 & $4,747,418$ \\
\hline 2011 & 17,605 & 2011 & 11,162 & 63.4 & 202.70 & 242.88 & 267.65 & $2,710,971$ \\
\hline 2012 & 8,874 & 2012 & 5,900 & 66.5 & 242.45 & 241.32 & 261.77 & $1,423,779$ \\
\hline 2013 & 3,540 & 2013 & 2,197 & 62.1 & 246.77 & 278.87 & 282.85 & 612,668 \\
\hline \multicolumn{8}{|c|}{ Total MF+CP } & $18,466,990$ \\
\hline \multicolumn{9}{|c|}{ Dispensed by Pharmacies } \\
\hline $\begin{array}{l}\text { MF } \\
(2011-2013)\end{array}$ & 7,111 & $\begin{array}{c}\text { MF } \\
\text { (2011-2013) }\end{array}$ & 4,002 & 56.3 & 92.88 & 155.24 & 200.72 & 621,277 \\
\hline 2011 & 1,698 & 2011 & 1,129 & 66.5 & 97.11 & 169.29 & 231.40 & 191,127 \\
\hline 2012 & 2,357 & 2012 & 1,427 & 60.5 & 102.96 & 147.34 & 168.35 & 210,260 \\
\hline 2013 & 3,056 & 2013 & 1,446 & 47.3 & 80.59 & 152.07 & 203.86 & 219,889 \\
\hline $\begin{array}{l}\text { CP } \\
(2011-2013)\end{array}$ & 453 & $\begin{array}{c}C P \\
(2011-2013) \\
\end{array}$ & 223 & 49.2 & 307.82 & 272.07 & 255.38 & 60,672 \\
\hline 2011 & 225 & 2011 & 78 & 34.7 & 314.40 & 296.06 & 236.42 & 23,093 \\
\hline 2012 & 175 & 2012 & 112 & 64.0 & 307.82 & 255.96 & 225.04 & 28,668 \\
\hline 2013 & 53 & 2013 & 33 & 62.3 & 46.79 & 270.03 & 374.11 & 8,911 \\
\hline \multicolumn{8}{|l|}{ Total MF+CP } & 681,948 \\
\hline \multicolumn{9}{|c|}{ Total MF+CP All Dispensed } \\
\hline Billed $^{\mathrm{h}}$ & 151,107 & & Reimbursed $^{\mathrm{i}}$ & 95,538 & & & & $19,148,938$ \\
\hline $\begin{array}{l}\text { a Number of pre } \\
{ }^{b} \text { Number of pre } \\
{ }^{c} \text { Percentage of } p \\
{ }^{d} \text { Median reimbr } \\
{ }^{e} \text { Mean reimburs } \\
\text { fStandard devia } \\
\text { gTotal reimburs } \\
{ }^{h} \text { Total number } \\
{ }^{i} \text { Total number o } \\
C P=\text { convenien }\end{array}$ & $\begin{array}{l}\text { tions billed (presc } \\
\text { tions reimbursed. } \\
\text { iptions reimburse } \\
\text { amount per presc } \\
\text { mount per prescri } \\
\text { from the mean. } \\
\text { nount in U.S. doll } \\
\text { dical food and cor } \\
\text { dical food and con } \\
\text { ckage; } \mathrm{MF}=\text { medic }\end{array}$ & $\begin{array}{l}\text { ptions claimed). } \\
\text { out of total num } \\
\text { iption billed (pre. } \\
\text { tion billed (presci } \\
\text { rs. } \\
\text { enience packages } \\
\text { enience packages } \\
\text { l food. }\end{array}$ & $\begin{array}{l}r \text { of prescriptions } \\
\text { riptions claimed). } \\
\text { tions claimed). }\end{array}$ & illed (prescription & laimed). & & & \\
\hline
\end{tabular}

the mean amount reimbursed per CP prescription was lower when physician-dispensed $(\$ 246.50, \mathrm{SD}=267.89)$ than when pharmacy-dispensed $(\$ 272.07, \mathrm{SD}=244.38)$.

The yearly mean amount reimbursed per prescription fluctuated for both physician-dispensed and pharmacy-dispensed MFs and CPs. Physician-dispensed amount reimbursed for MFs fluctuated from $\$ 195.64$ (SD = 206.96) in 2011 to $\$ 196.21(\mathrm{SD}=197.11)$ in 2012 to $\$ 173.27(\mathrm{SD}=205.05)$ in 2013 , and CP fluctuated from $\$ 242.88(S D=267.65)$ in 2011 to $\$ 241.32(\mathrm{SD}=261.77)$ in 2012 to $\$ 278.87(\mathrm{SD}=282.85)$ in 2013. Pharmacy-dispensed MFs also fluctuated from $\$ 169.29$ $(\mathrm{SD}=231.40)$ in 2011 to $\$ 147.34(\mathrm{SD}=168.35)$ in 2012 to $\$ 152.07$ (SD $=203.86)$ in 2013 , and CPs fluctuated from $\$ 296.06(S D=236.42)$ in 2011 to $\$ 255.96(S D=225.04)$ in 2012 to $\$ 270.03(\mathrm{SD}=374.11)$ in 2013.
While the yearly mean amount reimbursed per prescription fluctuated, the total reimbursed cost of physician-dispensed MFs (from approximately $\$ 6.69$ million in 2011 to $\$ 4.02$ million in 2012 to $\$ 3.01$ million in 2013) and CPs (from $\$ 2.71$ million in 2011 to $\$ 1.42$ million in 2012 to $\$ 0.61$ million in 2013) had decreased since the implementation of $A B 378$. This trend provided additional support for the effectiveness of $A B 378$ in regulating $\mathrm{MF}$ and $\mathrm{CP}$ use.

There was a different pattern of reimbursement for pharmacy-dispensed MFs and CPs, which was not regulated by AB 378. The total reimbursed cost of pharmacy-dispensed MFs increased from approximately $\$ 191,127$ in 2011 to $\$ 210,260$ in 2012 to $\$ 219,889$ in 2013 . The total yearly cost of pharmacydispensed CPs fluctuated from $\$ 23,093$ in 2011 to $\$ 28,668$ in 
Use and Costs of Medical Foods and Convenience-Packaged Drugs in the California Workers' Compensation System

TABLE 4 T-Tests Examining Difference in Mean of Reimbursed Amount Pre-AB 378 and Post-AB 378

\begin{tabular}{|c|c|c|c|c|c|c|c|c|c|c|c|}
\hline & & Prescriptions & $\begin{array}{l}\text { Mean Paid } \\
\text { Amount, \$ }\end{array}$ & $\begin{array}{c}\text { Standard } \\
\text { Deviation }\end{array}$ & $\mathrm{t}$ & df & $P$ Value & $\begin{array}{c}\text { Mean } \\
\text { Difference, } \$\end{array}$ & $\begin{array}{l}\text { Standard } \\
\text { Error }\end{array}$ & \multicolumn{2}{|c|}{$95 \% \mathrm{CI}$} \\
\hline \multicolumn{12}{|c|}{ Dispensed by Physicians } \\
\hline & Pre-AB 378 & 34,192 & 195.64 & 206.96 & & & & & & & \\
\hline \multirow[t]{3}{*}{$\mathrm{MF}$} & & & & & 6.54 & 72,052 & $<0.001$ & 9.95 & 1.52 & 6.97 & 12.93 \\
\hline & Post-AB 378 & 37,862 & 185.68 & 201.12 & & & & & & & \\
\hline & Pre-AB 378 & 11,162 & 242.88 & 267.65 & & & & & & & \\
\hline \multirow[t]{2}{*}{$\mathrm{CP}$} & & & & & -2.21 & 19,257 & 0.027 & -8.63 & 3.91 & -16.30 & -0.97 \\
\hline & Post-AB 378 & 8,097 & 251.51 & 268.16 & & & & & & & \\
\hline \multicolumn{12}{|c|}{ Dispensed by Pharmacies } \\
\hline & Pre-AB 378 & 2,556 & 157.04 & 198.94 & & & & & & & \\
\hline \multirow[t]{3}{*}{ MF } & & & & & 0.75 & 4,000 & 0.452 & 4.97 & 6.61 & -7.98 & 17.92 \\
\hline & Post-AB 378 & 1,446 & 152.07 & 203.86 & & & & & & & \\
\hline & Pre-AB 378 & 190 & 272.42 & 230.01 & & & & & & & \\
\hline \multirow[t]{2}{*}{$\mathrm{CP}$} & & & & & 0.05 & 221 & 0.961 & 2.39 & 48.27 & -92.74 & 97.52 \\
\hline & Post-AB 378 & 33 & 270.03 & 374.11 & & & & & & & \\
\hline
\end{tabular}

2012 to $\$ 8,911$ in 2013. Combining these trends with the fact that CAWCS was reimbursing a lower percentage of pharmacydispensed MFs and reimbursing a higher percentage of CPs each year from 2011 to 2013 suggests that there was a shift in dispensing behavior.

The effect of $\mathrm{AB} 378$ on regulating physician-dispensed MFs and CPs was explored. For physician-dispensed MFs, the mean amount reimbursed per prescription was $\$ 195.64$ in the pre-AB 378 group and $\$ 185.68$ in the post-AB 378 group (Table 4). The mean amount reimbursed decreased by $\$ 9.95$, with a t-test result indicating that the difference in mean amount reimbursed was statistically significant $(P<0.001)$. This result provided evidence that $A B 378$ was associated with a significant reduction in the reimbursed amount for physician-dispensed MFs.

For physician-dispensed CP prescriptions, the difference in mean amount reimbursed ( $\$ 242.88$ in the pre-AB 378 years and $\$ 251.51$ in the post-AB 378 years) increased by $\$ 8.63$, with a t-test result indicating that the difference was statistically significant $(P=0.027)$. While AB 378 had an effect on the cost of MFs, the cost of CPs per prescription continued to rise. The statistically significant increase in the mean amount reimbursed for CP was not as hypothesized.

For pharmacy-dispensed MF prescriptions, the difference in mean amount reimbursed per MF claim (\$157.04 in the pre-AB 378 group and $\$ 152.07$ in the post-AB 378 group) was $\$ 4.97$, with a t-test indicating that the difference was not statistically significant $(P=0.452)$. The t-test for pharmacy-dispensed CP prescriptions demonstrated that the difference in mean amount reimbursed per CP prescription (\$272.42 in the pre-AB 378 group and $\$ 270.03$ in the post-AB 378 group) was $\$ 2.39$ $(P=0.961)$. The results from t-tests showed no statistically significant difference in mean amount reimbursed for MFs and
CPs to pharmacies before and after $\mathrm{AB}$ 378; the finding was expected and as hypothesized because $\mathrm{AB} 378$ did not regulate pharmacy-dispensed MFs and CPs.

\section{Discussion}

This study is the first to examine the effect of $A B 378$ on the use of MFs and CPs from 2011 to 2013 and compare the differences in utilization volume and cost between different dispensers. The investigation of the pattern of MF and CP use and costs for physician-dispensed MFs before and after passage of $A B$ 378 found that this legislation had the desired effect of lowering physician prescription of these products. The results found that although MFs and CPs accounted for only $1.2 \%$ of all billed prescriptions (claims) in CAWCS between 2011 and 2013, physicians dispensed almost all (95\%) of those MFs and CPs, which resulted in $\$ 19$ million reimbursed in the 3-year period. The most frequently billed MFs and CPs were for indications of insomnia and pain, which would be expected for medication claims in worker's compensation systems.

Both the significant cost and the pattern of use are consistent with the findings in previous studies, which first raised the possible conflict of physicians both prescribing and dispensing MFs and CPs. ${ }^{4}$ This high physician prescription volume is especially concerning because the necessity for and evidence of safety and effectiveness of non-IEM MFs are often also lacking. ${ }^{2}$ This investigation also raised questions about how MFs and CPs - which are not allowed to have NDC numbers-should be priced and paid for, given that most medication billing systems (as does CAWCS) require an NDC number.

The investigation demonstrated that physician-dispensed MFs were costlier than pharmacy-dispensed MFs. The findings are congruent with the current literature, which demonstrated that physicians dispensed 2.99 times more products than 
pharmacies and that the medical cost for patients receiving physician-dispensed medications was 39\% higher than when patients were not receiving physician-dispensed medications. ${ }^{8}$ Nevertheless, the number of physician-dispensed MFs experienced a downward trend from 2011 to 2013, whereas the number of pharmacy-dispensed MFs fluctuated.

The changes in number of MFs billed and reimbursed may be indicative that $\mathrm{AB} 378$ exerted the desired effect on physician prescribing behavior. The lack of change in rate of reimbursed physician-dispensed MF prescriptions indicated that physicians curbed their prescription numbers without needing CAWCS to deny more claims. The pattern also suggested that a spillover effect existed, in which the nonregulated pharmacies were dispensing more MFs because patients might not have been able to get MF and CP prescriptions from physicians. In response to the spillover effect, the analysis showed that a higher percentage of pharmacy-dispensed MF claims were denied by CAWCS.

To gain further insight on physicians' prescribing behavior, we examined the amount that CAWCS paid for MFs and $\mathrm{CPs}$. While the yearly mean amount reimbursed per prescription fluctuated for both physician-dispensed and pharmacydispensed MFs and CPs, the difference in cost per MF prescription before and after the implementation of $A B 378$ was statistically significant, which provided further support for the positive effect of $A B 378$ in regulating MF use. In addition, the total cost of physician-dispensed MFs, regulated by AB 378, decreased steadily.

The analysis also found additional evidence for a spillover effect, in which the total yearly cost of pharmacy-dispensed MFs increased steadily while the total yearly cost of CPs fluctuated. The spillover effect suggested that CAWCS and others seeing rapid expansion of MF use should also move to regulate pharmacy-dispensed non-IEM MFs and CPs to contain overprescribing and overbilling of these products, especially with unproven safety and effectiveness.

The results presented here provide some evidence that AB 378 exerted regulatory effect on physicians' behavior in dispensing MFs but not in dispensing CPs. The change in physician behavior also affected how pharmacies dispensed MFs and CPs. The percentage of MF and CP use may be small relative to overall drug prescriptions, but these products were still costly to CAWCS. AB 378 curbed prescription frequency and cost for physician-dispensed MFs and CPs, but prescription frequency for pharmacy-dispensed MFs, unregulated by AB 378, was still rising.

While legislation such as AB 378 can exert an effect on MF and $\mathrm{CP}$ use, it is important also to pay attention to the effect of new emerging MFs and CPs and how these new products may affect overall use of MFs and CPs and the effectiveness of regulations. For example, analysis showed that a new product Somnicin became available in 2012 and immediately became the most frequently reimbursed pharmacy-dispensed product in 2012 and 2013. The product contains melatonin, 5-hydroxytryptophan, L-tryptophan, vitamin B6, and magnesium. Since 5-hydroxytryptophan is identified as an MF, Somnicin is classified as an MF despite the fact that the manufacturer does not categorize Somnicin as an MF. ${ }^{9}$ Concomitantly, there are no scientific studies supporting the efficacy of Somnicin.

The Office of Disability Guidelines does not recommend the use of Somnicin for pain-induced insomnia. ${ }^{10} \mathrm{~A}$ warning letter from an independent medical review concluded, based on the California Medical Treatment Utilization Schedule, that Somnicin is neither medically necessary nor appropriate for the treatment of pain-induced insomnia because the condition lacks distinctive nutritional requirements as required for an MF. Somnicin therefore violates the FDA guideline for MFs and should not be reimbursed as an MF by CAWCS. However, due to current federal and state regulation, Somnicin is an MF and accounts for 36\% of overall pharmacy-dispensed MFs.

\section{Limitations}

While this study took the first step in examining the effect of $\mathrm{AB} 378$, the analyses inevitably had several limitations. First, due to the absence of a list of MFs available, there was no starting standard, leaving room for potential misclassification. To remedy this issue, NDC numbers, packaging information, MF ingredients, and RED BOOK information were evaluated to ensure the accurate classification and incorporation of all MFs and CPs in the dataset.

Second, as with many claims datasets, there were some missing data, duplications, and potential claims errors. The service adjustment code was used to identify duplications and errors and decide if each empty cell was empty due to missing data or simply a zero for billed, reimbursed (paid), denied, or adjusted amount. For example, if an entry was coded as denied by CAWCS, it was assumed that the total amount reimbursed was 0 , even if a reimbursed amount was shown. Conversely, if a reimbursed amount was 0 , but the service adjustment code indicated that the prescription was reimbursed, the prescription was categorized as paid.

In many cases, the reimbursed amount was 0 because the reimbursement for the prescription line (claim) was included with the reimbursement for the other line. The authors recognized that the 0 entries could sometimes be an indication of error but chose to assume that the service adjustment code provided the most updated information on the claim reimbursement process.

Some entries were missing both drug name and NDC number or contained an invalid NDC number. These products could not be identified or confirmed so ( $0.4 \%$ of all entries) were dropped and likely did not significantly bias our findings due to their small number. In the physician-dispensed dataset, a majority of the claims were missing billed amounts, so these were not reported. Reimbursed amounts were-a more accurate reflection of costs to the claims system, and so they were analyzed for the physician-dispensed analysis. 
Finally, the analysis is only the first step to examining the full regulatory effect of $A B 378$. T-tests were used to examine the difference in cost pre-AB 378 and post-AB 378, but this method did not account for other confounding variables, which the analyses were unable to control for due to the limitation of available variables of interest. It would be beneficial for future analyses to control for additional factors.

Despite these limitations, this study contributed to the understanding of non-IEM MF and $\mathrm{CP}$ use for the general population by highlighting the effect of a WC system-specific reimbursement policy and how the policy can curb MF and $\mathrm{CP}$ use. The value of MFs and CPs used in patients in WC systems is questionable, given their high costs and lack of robust evidence for efficacy, as reported in 2 previous studies. ${ }^{2,4}$ Wilson et al. (2018) found that most studies on non-IEM MFs were conducted without scientific rigor ${ }^{2}$; that is, these studies did not employ randomized controlled trials, lacked sufficient sample size, or did not discuss safety and efficacy. Although not addressed specifically here, MFs may also have safety problems, since there is not oversight as to the quality and consistency of their ingredients.

\section{Conclusions}

The results of this study demonstrated that MFs dispensed by physicians, which have been regulated by $\mathrm{AB} 378$ since January 2012, experienced a reduction in mean amount reimbursed compared with the mean amount reimbursed in 2011. Legislative measures, such as AB 378 in California, may partly explain the pattern; however, there is a need to begin compiling consistent data on the availability, quality, and need for MFs generally as well as factors influencing MF prescriptions to fully understand the variation in MF use.

Given the rapidly expanding market and clear pricing incentives, the use of MFs and CPs can increase overall and in WC systems-especially because there is little or no oversight on the use or pricing of these products. Other states should look at their own WC systems to evaluate the extent of potentially unrecognized use and costs attributed to MFs and CPs. There is a need for a better understanding of the use of these products within other states' WCs. Future policy efforts should continue to monitor the use and costs of non-IEM MFs as well as focus on how WC claim systems can stay abreast of the need for and safety of non-IEM MFs in a rapidly evolving industry.

\section{DISCLOSURES}

Funding for this study was contributed by the California Workers' Compensation System. The authors have nothing to disclose.

\section{REFERENCES}

1. U.S. Food \& Drug Administration. Frequently asked questions about medical foods; second edition. Guidance for industry. May 2016. Available at: https://www.fda.gov/downloads/Food/GuidanceRegulation/ GuidanceDocumentsRegulatoryInformation/UCM500094.pdf. Accessed June 11, 2018.

2. Wilson L, Lin TK, Oh A, Cao V. A gray area for reimbursement: medical foods for non-inborn errors of metabolism. Am J Manag Care. Forthcoming July 2018.

3. Stephens G. Business insights: the emerging market for medical foods. September 1, 2012. Available at: http://www.nutraceuticalsworld.com/ issues/2012-09/view_columns/business-insights-the-emerging-market-formedical-foods. Accessed June 11, 2018

4. Wynn BO. Use of compound drugs, medical foods, and co-packs in California's workers' compensation program: an overview of the issues. Prepared for the Commission on Health, Safety and Workers' Compensation. WR-828-CHSWC. RAND Center for Health and Safety in the Workplace. January 2011. Available at: https://www.rand.org/pubs/working_papers/ WR828.html. Accessed June 11, 2018

5. Ireland J, Swedlow A. The cost and utilization of compound drugs, convenience packs and medical foods in California workers' compensation. California Workers' Compensation Institute. CWCI Research Notes. August 2010. Available at: https://www.cwci.org/document.php?file=1337. pdf. Accessed June 11, 2018

6. Pullon R. An introduction to the medical foods industry. May 24 2013. Available at: https://www.scribd.com/document/230447302/ An-Introduction-to-the-Medical-Foods-Industry. Accessed June 11, 2018

7. State of California Legislative Counsel. California Assembly Bill 378. Available at: http://www.leginfo.ca.gov/pub/11-12/bill/asm/ab_0351-0400/ ab_378_bill_20110509_amended_asm_v97.html. Accessed June 11, 2018.

8. White JA, Tao X, Artuso RD, Bilinski C, Rademacher J, Bernacki EJ. Effect of physician-dispensed medication on workers' compensation claim outcomes in the state of Illinois. J Occup Environ Med. 2014;56(5):459-64.

9. Maximus Federal Services. Independent medical review final determination letter. December 24, 2013. Available at: http://dir.ca.gov/dwc/IMR/ IMR-Decisions/IMR-Decisions/IMR\%20Decisions/IMR2013_10001-20000/ CM13-0018109.pdf. Accessed June 11, 2018.

10. Texas Department of Insurance. Medical fee dispute resolution findings and decision. December 9, 2015. Available at: http://www.tdi.texas.gov/medcases/medfee15/m4152935.pdf. Accessed June 11, 2018.

\section{Authors}

TRACY KUO LIN, MSc, PhD, and LESLIE WILSON, PhD,

School of Pharmacy, University of California, San Francisco.

AUTHOR CORRESPONDENCE: Tracy Kuo Lin, MSc, PhD, 533 Parnassus Ave., U12-A, University of California, San Francisco, San Francisco, CA 94143. Tel.: 415.502.6429; E-mail: tracy.lin@ucsf.edu. 